\title{
Effectiveness of scoliosis-specific exercises for alleviating adolescent idiopathic scoliosis: a systematic review
}

\author{
Yunli Fan ${ }^{1,2,3}$, Qing Ren ${ }^{3}$, Michael Kai Tsun To ${ }^{1,3}$ and Jason Pui Yin Cheung ${ }^{1,3^{*}}$ (D)
}

\begin{abstract}
Background: Adolescent idiopathic scoliosis (AIS) is the most common pediatric spinal deformity with reported complications including pain, mental health concern and respiratory dysfunction. The scoliosis-specific exercise (SSE) is prescribed throughout pubertal growth to slow progression although effects are unclear. This review aims to establish the effectiveness of SSE for alleviating AIS in terms of reducing Cobb angle, improving trunk asymmetry and quality of life (QoL). Additionally, it aims to define the effects of age, skeletal maturity, curve magnitude and exercise compliance on the outcomes of SSE.

Methods: A systematic reviewed was conducted to net SSE articles. Searched databases included PubMed, MEDL INE, Cochrane Library, Scopus, CINAHL and Google scholar. The quality of study was critically appraised according to the PEDro scale.

Results: A total of ten trials with an average PEDro score of 6.9/10 were examined in this study. Two randomized controlled trials (RCTs) and two clinical controlled trials suggested that SSE alone and with bracing or traditional exercise had clinical significance in reducing Cobb angle more than $5^{\circ}$. One RCT specifically implicated no comparable effects between bracing and SSE in prevention of curve progression for moderate scoliosis. There was insufficient evidence to support the positive effects of SSE on improving truck asymmetry $(n=4)$ and QoL $(n=3)$. Five studies evaluated the interaction effects of age $(n=2)$, skeletal maturity $(n=1)$ and curve magnitude $(n=2)$ with SSE in reducing Cobb angle yet without drawing any firm conclusions.
\end{abstract}

Conclusions: Insufficient evidence is available to prove that SSE with or without other conservative treatments can reduce Cobb angle, improve trunk balance and QoL. The interaction effects of age, skeletal maturity, curve magnitude, and exercise compliance with SSE in reducing Cobb angle are not proven. Future studies should investigate the relationship of influencing factors and SSE in treating AIS but not only testing its effectiveness.

Trial registration: INPLASY202050100.

Keywords: Adolescent idiopathic scoliosis, Scoliosis specific exercise, Cobb angle, Truncal asymmetry, Quality of life

\footnotetext{
* Correspondence: cheungjp@hku.hk

'Department of Orthopaedics and Traumatology, The University of Hong Kong - Shenzhen Hospital, 1 Haiyuan 1st Road, Futian District, Shenzhen, Guangdong, China

${ }^{3}$ Department of Orthopaedics and Traumatology, The University of Hong Kong, 5/F Professional Block, Queen Mary Hospital, Pokfulam, Hong Kong SAR, China

Full list of author information is available at the end of the article
}

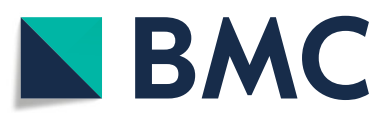

( The Author(s). 2020 Open Access This article is licensed under a Creative Commons Attribution 4.0 International License, which permits use, sharing, adaptation, distribution and reproduction in any medium or format, as long as you give appropriate credit to the original author(s) and the source, provide a link to the Creative Commons licence, and indicate if changes were made. The images or other third party material in this article are included in the article's Creative Commons licence, unless indicated otherwise in a credit line to the material. If material is not included in the article's Creative Commons licence and your intended use is not permitted by statutory regulation or exceeds the permitted use, you will need to obtain permission directly from the copyright holder. To view a copy of this licence, visit http://creativecommons.org/licenses/by/4.0/. The Creative Commons Public Domain Dedication waiver (http://creativecommons.org/publicdomain/zero/1.0/) applies to the data made available in this article, unless otherwise stated in a credit line to the data. 


\section{Background}

Adolescent idiopathic scoliosis (AIS), characterized by lateral deviation, axial rotation, and abnormal sagittal curvature of the spine, is the most common (70-80\%) spinal deformity with unclear etiology [1]. Its prevalence is approximately $0.47-5.2 \%$ in the general adolescent population [2]. This condition may lead to cosmetic concerns [3], pain [4], and respiratory dysfunction [5]. AIS was reported in almost $10 \%$ of patients requiring either conservative or surgical treatment [6]. Surgery is reserved for severe curves of $50^{\circ}$, whereas bracing and scoliosis-specific exercise (SSE) are reserved for mild $\left(10^{\circ}-25^{\circ}\right)$ and moderate $\left(25^{\circ}-45^{\circ}\right)$ curves to prevent progression to the operative stage [7].

Bracing is the most common conservative treatment if the Cobb angle is $>25^{\circ}$ in patients with growth potential [8-10]. It produces an external pushing force to straighten the trunk and to derotate the rib cage. Skeletal maturity [8], in-brace correction [11], curve magnitude [12], flexibility [13-15] and compliance with brace wearing [16] are significant factors influencing the outcomes of brace treatment. However, bracing can be stressful for patients, induce a flatter back [17], and negatively affect quality of life (QoL) [18-23]. Additionally, most braces are uncomfortable to wear, resulting in poor brace-wearing compliance [24]. In contrast, SSE is commonly accepted by patients [25]. Moreover, SSE is recommended alone or as an add-on to bracing for preventing scoliosis progression [26]. Several techniques of SSE have been established in previous studies [27-32]; some techniques are described more often than others [33]. Although the method used varies, all techniques adhere to the same principle, namely: 1) three-dimensional self-correction; 2) training activities of daily living; and 3) stabilization of corrected postures [34]. Updated studies have reported promising effects of SSE on curve regression [35-48], which warrants a thorough investigation.

The latest review concluded that no valid evidence proved the effect of SSE on curve progression prevention [49]. In particular, three studies have used the same cohort [43-45]; one was a single-arm prospective study without a comparative untreated group [42]. Regarding the National Health and Medical Research Council (NHMRC) hierarchy of evidence [50], a randomized control trial (RCT) is considered the best methodology for answering intervention questions in a literature review. Thus, the quality of enrolled studies in that review was relatively poor [49]. Another three systematic reviews enrolled studies between 2005 and 2017 and found insufficient and low-quality clinical trials showing effects of SSE on improving the scoliotic deformity [33, 51, 52]. One review confirmed the promising effects of the
Schroth method in curve regression but had analyzed only four studies [51]; one review analyzed nine articles of which three (33\%) did not use SSE and one (11\%) was an outdated article published 15 years ago [33]. One study analyzed eight articles of which three (37.5\%) were rated as being low quality (PEDro score: 3 ), one (12.5\%) had a retrospective study design, and $50 \%(n=4)$ were published 10 years ago [52]. A number of controlled trials have been published after 2017, which calls for an updated systematic review.

Based on currently available evidence, SSE may be effective for improving spinal deformity; however, this is supported by only low-quality evidence. Moreover, unlike bracing, no review discussed the influencing factor of SSE on scoliotic curvature improvement. Understanding how SSE functions is crucial rather than accepting its effectiveness. Therefore, this review aims to access the most updated SSE studies that adhered to the Society on Scoliosis Orthopedic and Rehabilitation Treatment (SOSORT) exercise principle [34] to evaluate the effect of SSE on scoliotic deformity improvement. Moreover, we aim to define the effects of age, skeletal maturity, curve magnitude, and exercise compliance on SSE outcomes.

\section{Methods}

\section{Search strategy}

This systematic review replicated the search strategy adopted by the Cochrane Review from January 1, 2010 to February 29, 2020 in the following six databases: PubMed, MEDLINE, Cochrane Library, Scopus, CINA HL, and Google Scholar (Fig. 1). Key search items consisted of "AIS", or "idiopathic scoliosis", and "exercise", or "scoliosis specific exercise", or "physiotherapy", or "Schroth", or "SEAS", or "DoboMed, or "Side-shift" or "FITs" or "randomi" or "placebo" or "control"". These included subject headings, text words, methodological terms, disorder terms, and treatment terms, and all are listed in full in the search strategy in Additional file 1. This review protocol was registered on the INPLASY.COM with registration number INPLASY202050100. Searched results from each database were cross-checked by two independent researchers. Potentially relevant abstracts were screened based on the inclusion criteria, and full-text articles were obtained for eligible results. The two researchers discussed any disagreements regarding accepting full-text articles until consensus was achieved.

\section{Inclusion criteria}

The PICOS principle was applied to set the inclusion criteria, specifically described as: 1) $\mathrm{P}$ (population): adolescents with idiopathic scoliosis, 2) I (intervention): reported any of the SSE methods in either 


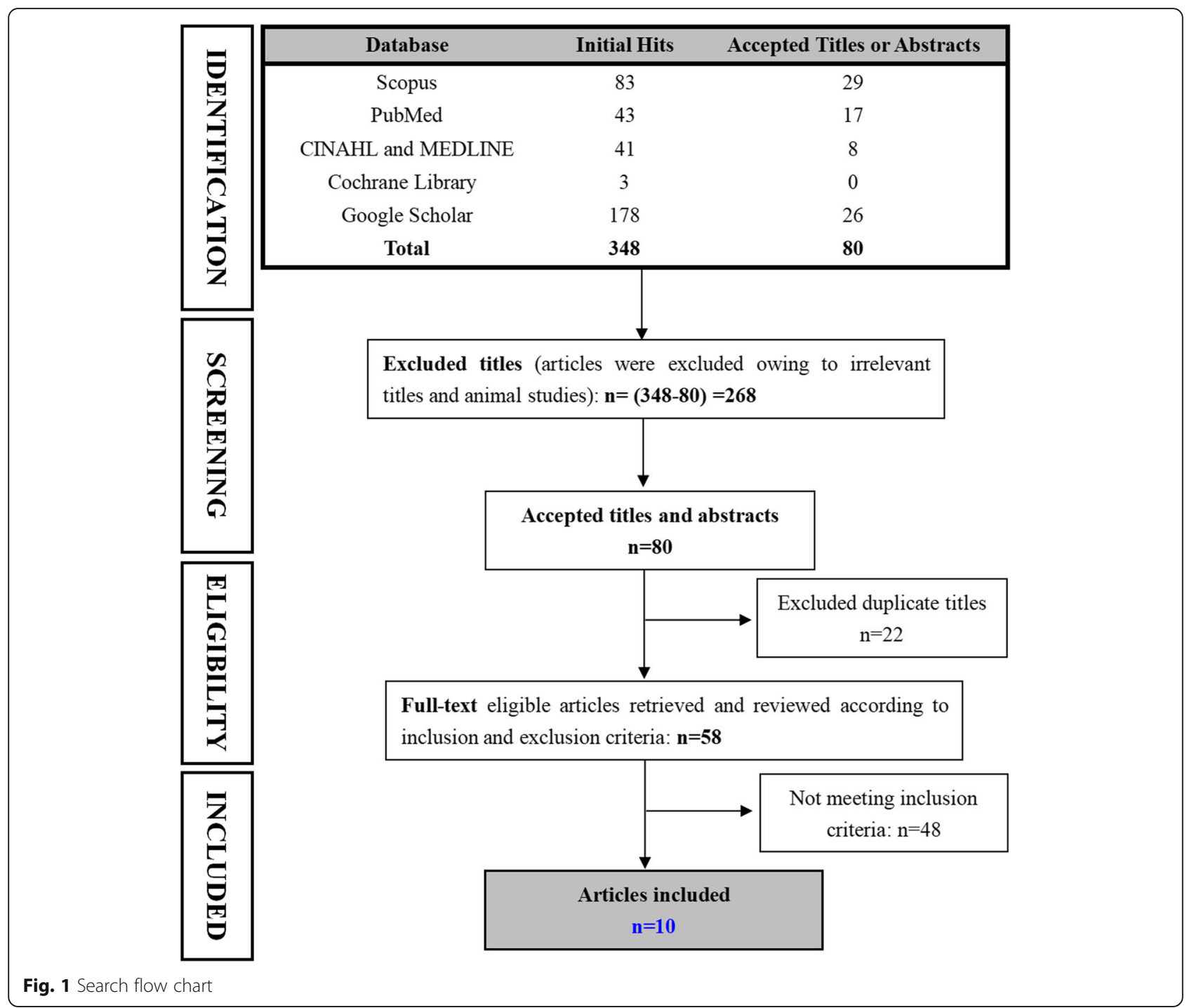

study or control group, 3) C (comparison): compared with traditional exercise, no treatment, standard care, brace, or any other non-SSE, 4) O (outcome): Cobb angle was reported in degrees as the primary outcome to evaluate effects on curve regression, with or without the secondary outcome defined as the truncal asymmetry (angle of trunk rotation in degrees: ATR) or condition-related function/QoL measured using validated questionnaires (e.g. 22-item or 23-item Scoliosis Research Society questionnaire), and 5) S (study design): prospective studies with controls that were published in or after 2010 were included.

\section{Exclusion criteria}

Any animal/pharmacological study, retrospective human clinical trial, or prospective single-arm study written in a language other than English or published before 2010 was excluded.
Evidence hierarchy and methodological appraisal

The NHMRC hierarchy of evidence was adopted to evaluate the evidence level [50]. Level II evidence (RCT) was considered the best methodology to answer intervention-related questions in a systematic review. However, considering the limited number of RCT in the most up-to-date reviews $[33,49,51,52]$, prospective clinical control trials (CCT: Level III) were also analyzed in this study.

Methodological qualities were measured using the PEDro scale [53]. The PEDro scale was proven to have validity and reliability for evaluating the methodological quality of clinical trials [53]. It has been commonly used to evaluate physiotherapy studies [54]. The PEDro scale scores methodology based on 10 items: 1) random allocation, 2) concealed allocation, 3) similarity at baseline, 4) subject blinding, 5) therapist blinding, 6) assessor blinding, 7) > 85\% follow-up for at least one outcome, 8 ) 
intention-to-treat analysis, 9) between-group comparison for at least one outcome, and 10) point and variability measures for at least one outcome (Table 1). Items were scored as either present [1] or absent (0). A score out of 10 ranked the study as having weak (PEDro score: $0-4$ ), moderate (PEDro score: $5-7$ ), and strong (PEDro score: $8-10)$ quality.

\section{Results}

\section{Search results}

A total of 348 initial hits were obtained from six databases. In total, 268 unrelated and 22 duplicate titles were excluded after their titles and abstracts were screened (Fig. 1). Up to 48 of 58 full-text articles were excluded because of the following reasons: 1 ) ineligible study designs (single-arm study: $n=8$, retrospective study: $n=5$, observational study: $n=2$, and feasible study: $n=3$ ); 2 ) incorrect interventions (head positioning: $n=1$, general stretching: $\mathrm{n}=5$, core muscle training: $n=7$, spinal manual therapy: $\mathrm{n}=3$, electrostimulation therapy: $n=2$, and traction: $\mathrm{n}=1)$; 3 ) inappropriate patient population $(\mathrm{n}=$ 3 , adult participants); 4) inappropriate outcomes ( $\mathrm{n}=2$, Cobb angle was not compared); 5) full-text was not in English $(n=4)$; and 6) duplicates $(n=2$, same cohort with multiple publications). Finally, 10 articles were included in this review (Fig. 1).

\section{Evidence hierarchy and methodological appraisal}

Eight articles with an RCT methodology (80\%) were classified as providing level II evidence [35-37, 41, 4548], and two articles with prospective CCT methodology (20\%) were classified as providing level III evidence [38, 40] (Table 1). The PEDro scale was ranked from 5 to 9 , with an average score of 6.9/10 for overall articles (Table 1). Specifically, the average scores of Schroth studies [35-38, 45], scientific exercise approach to scoliosis (SEAS) studies [41, 47, 48], and alternative SSE [40, 46] studies were $6.4(n=5$, score: $5-8), 8(n=3$, score: $8-9)$, and $6.5(n=2$, score: $6-7)$, respectively. Criteria 4 (blinding subjects) and 5 (blinding therapists) were not met for $80 \%(n=8)$ of studies [35-38, 45-48]. However, one RCT [41] reported participant blinding, whereas one CCT [40] reported therapist blinding.

\section{Characteristics of included studies}

Five trials adopted the Schroth method (Table 2). In particular, three RCTs compared the Schroth method alone with standard care [45], Pilates [35], and home exercise [37]; one CCT compared the Schroth method and bracing with bracing alone for moderate scoliosis [38]; one RCT compared the Schroth method and respiratory exercises with the Schroth method alone for mild to moderate scoliosis [36]. Three trials adopted the SEAS method (Table 2). These included one RCT comparing
SEAS with core stabilization exercises in patients with moderate scoliosis [47], one RCT comparing SEAS with bracing for moderate scoliosis [48], and one comparing SEAS with traditional exercises for mild scoliosis [41]. Another two studies adopted alternative SSE (body awareness, and Xinmiao approach) [40, 46]: one was an RCT comparing traditional exercises and body awareness exercises with traditional exercise alone for both mild and moderate scoliosis [46]; a CCT study grouped participants by age ( $<10$ years, $10-12$ years, and $13-15$ years) to determine the relationship of age, skeletal maturity, and gender with intervention effects [40].

Variations in intervention dosage were found, from daily to every other day (Table 2). Four trials reported > 1-year follow-up, whereas six trials had study periods of 2-6 months (Table 3). However, only five studies reported exercise compliance in percentage values of prescribed dosage (Table 3).

Six studies compared the truncal asymmetry pre- and post-intervention (Table 3). Four of them showed that SSE was not superior to core exercises, traditional exercises, and bracing for ATR improvement $[38,46,47]$ or shoulder balance [48]. Two studies showed better improvement of ATR in the study group [37, 41]. For QoL (Table 3), five studies adopted the SRS-22 questionnaire [38, 41, 46-48], and one study adopted the SRS-23 questionnaire [37]. However, a high initial score (mean score: 3.8-4.2) was noted in all studies (Table 3), with three studies reporting better QoL outcomes in terms of function and mental domain, favoring the SSE group [41, 46, 48]. Two studies found no significant differences of QoL between the groups in either adding SSE to bracing treatment or comparing supervised SSE with home exercises [37, 46]. Another study found improved pain domain outcomes in the core exercise group only [47].

\section{Proposed questions}

\section{Can SSE improve scoliotic deformity?}

Ten studies with 494 participants were enrolled in this review (Table 2). Five trials (three RCTs and two CCTs) with moderate study quality showed significant curve regression in terms of reducing Cobb angles beyond the measurement error of $5^{\circ}$ (Table 3). Three studies [35, 40, 41] enrolled participants with mild scoliosis (Cobb angle: $10^{\circ}-27^{\circ}$ ): Monticone et al. reported a decrease of $5.3^{\circ}$ with SEAS but an increase of $1.7^{\circ}$ with general exercise at skeletal maturity [41]; Kim et al. found a large curve regression from $23.6 \pm 1.5^{\circ}$ down to $12.0 \pm 4.7^{\circ}$ in the Schroth group, whereas a reduction from $24.0 \pm 2.6^{\circ}$ to $16.0 \pm 6.9^{\circ}$ was observed in the Pilates group after 3 months of exercises [35]; Liu et al. grouped participants according to age and revealed that notable curve 
Table 1 Evidence hierarchy and methodological appraisal

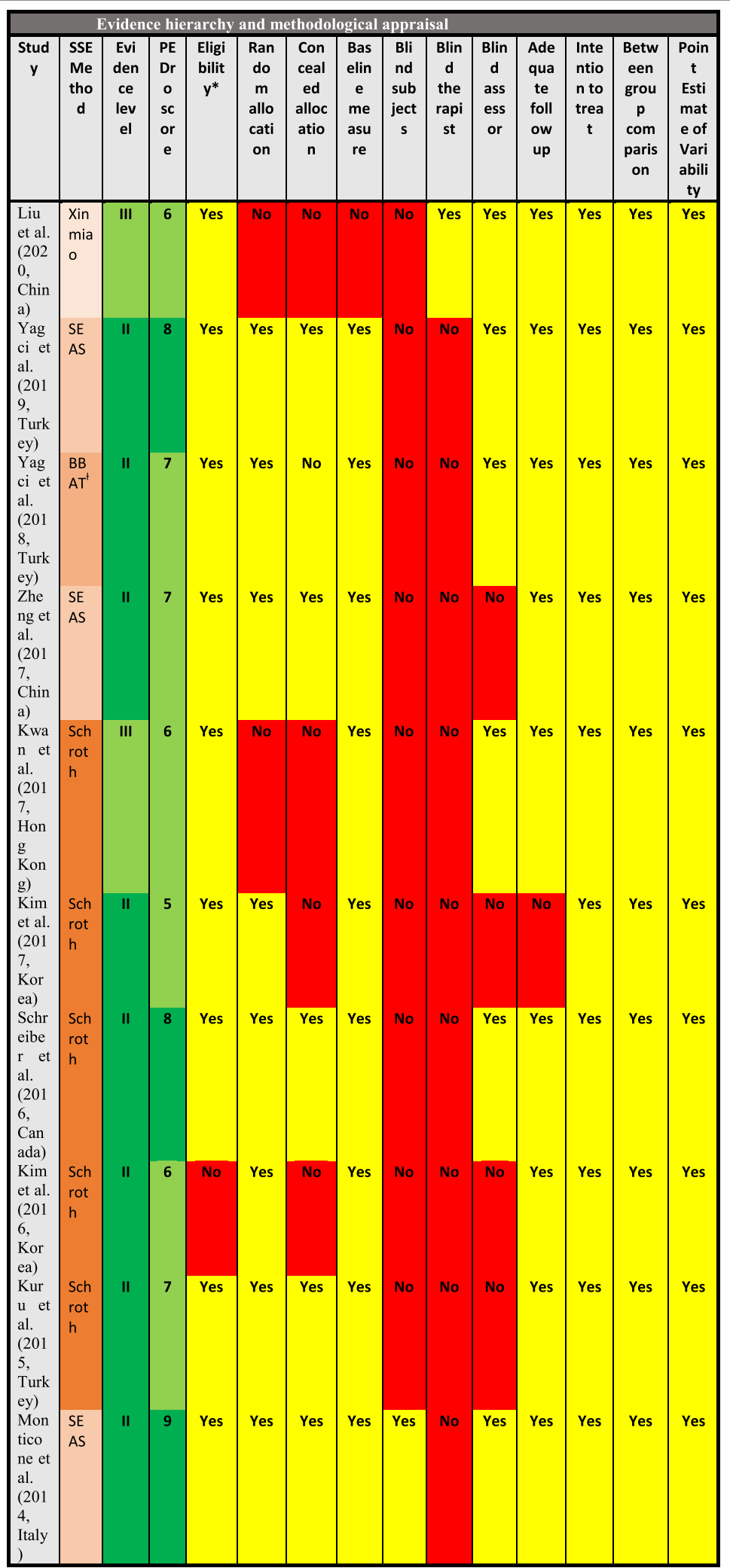




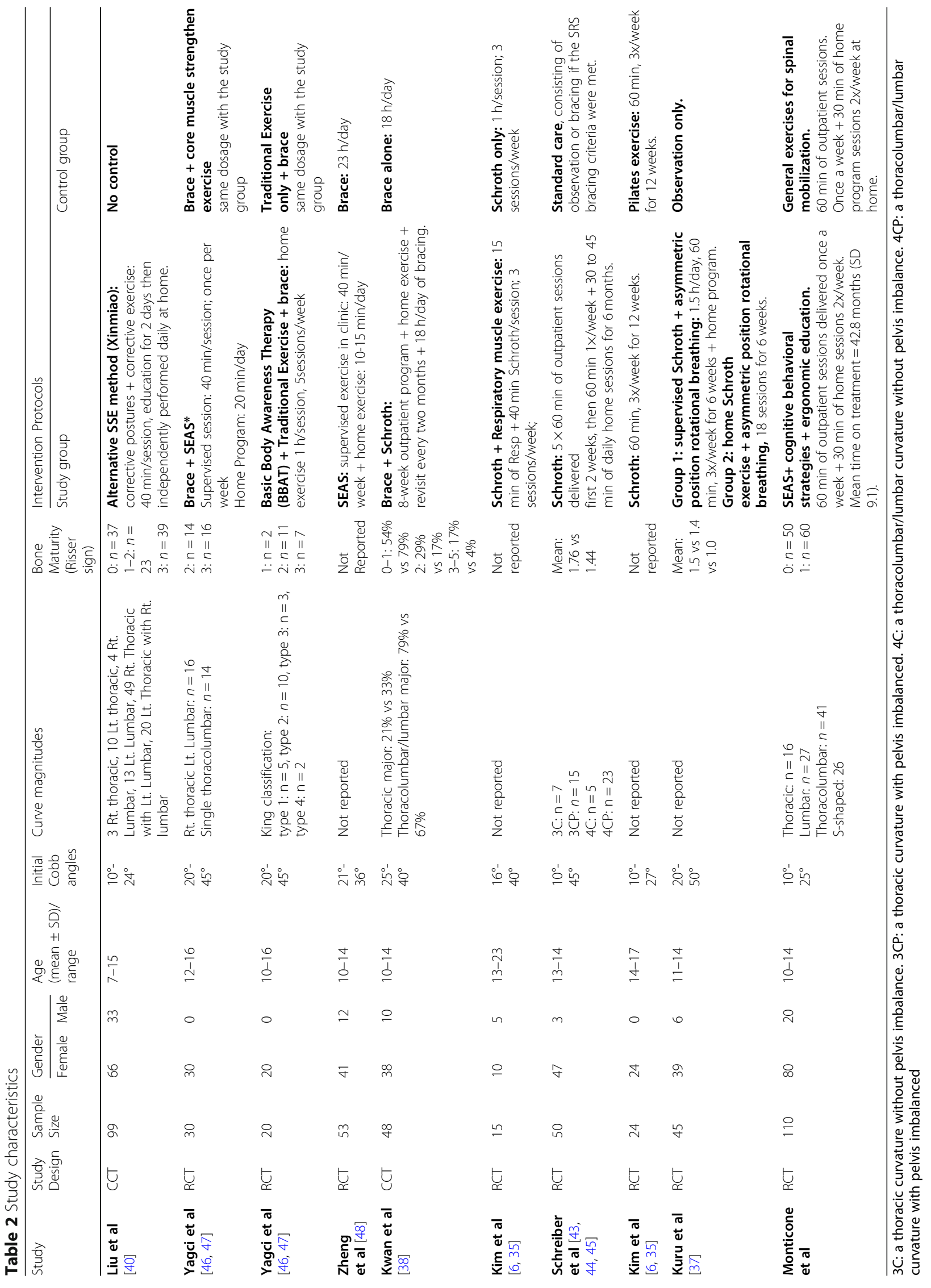




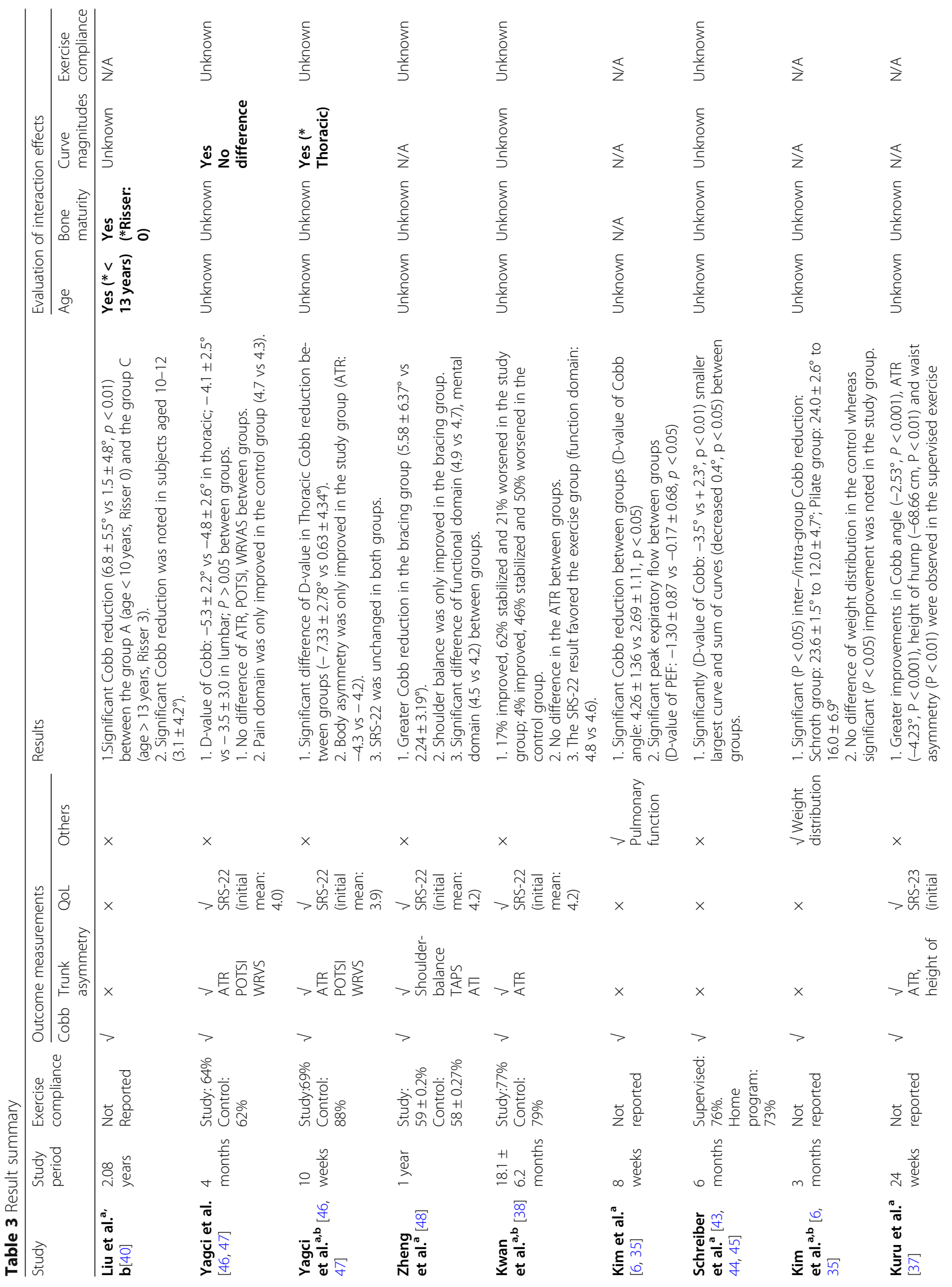




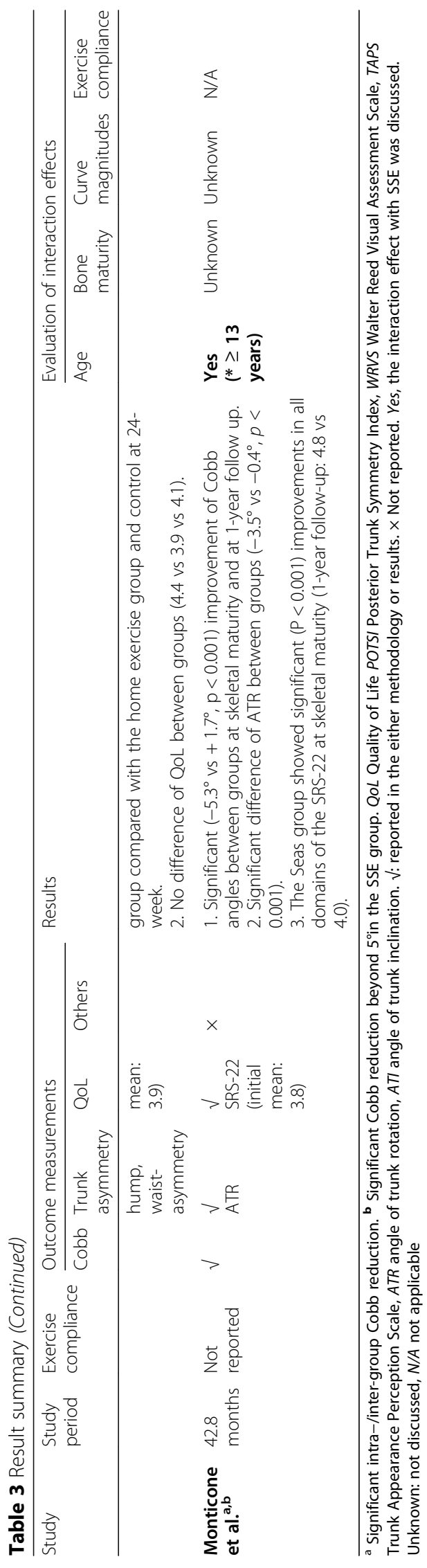


regressions were favored in patients younger than 13 years (a decrease of Cobb angle: $6.8 \pm 5.5^{\circ}$ for age $<10$ years; $3.1 \pm 4.2^{\circ}$ for age $10-12$ years) and with Risser stage 0 (a decrease of Cobb angle: $5.7 \pm 5.6^{\circ}$ ) at 2-year follow-up [40]. Another two studies involved bracewearing patients with moderate scoliosis $[38,46]$. Kwan et al. found that $17 \%$ of the participants showed improvement with Schroth exercises, whereas only $4 \%$ improved with no exercise at 1.5-year follow-up [38]. Yagci et al. adopted body awareness exercise with bracing and revealed a significant Cobb angle reduction $(-7.33 \pm$ $2.78^{\circ}$ vs $\left.0.63 \pm 4.34^{\circ}, p<0.05\right)$ of the thoracic curvature between the groups [46].

Three RCTs showed statistically significant reductions in Cobb angle, but differences were not of clinical significance (Table 3). Kim et al. conducted an 8-week-long study and reported a reduction of Cobb angle by $4.26 \pm$ $1.36^{\circ}$ after Schroth with respiratory exercises [36]. Schreiber et al. conducted a 6-month-long study and demonstrated a $3.5^{\circ}$ decrease in the largest curves but only a decrease of $0.4^{\circ}$ in the sum of curves (root mean square value) with Schroth therapy [45]. Kuru et al. performed a 6-month-long study with three groups and found greater Cobb angle reduction $\left(-2.53^{\circ}, p=0.03\right)$ in those who relied on supervised Schroth therapy [37].

Furthermore, two RCTs concluded that SSE was not superior than either bracing or core muscle exercises in improving scoliotic deformity (Table 3). Yagci et al. compared the SEAS with core muscle exercises in participants wearing a brace and revealed comparable effects between the thoracic $\left(-5.3 \pm 2.2^{\circ}\right.$ vs $\left.4.8 \pm 2.6^{\circ}\right)$ and lumbar $\left(-4.1 \pm 2.5^{\circ}\right.$ vs $\left.-3.5 \pm 3.0^{\circ}\right)$ curvatures [47]. Zheng et al. compared the SEAS alone with bracing for moderate scoliosis and suggested that a notable reduction in Cobb angle only favored the bracing group (bracing: $5.58 \pm 6.37^{\circ}$ vs SEAS: $2.24 \pm 3.19^{\circ}, p=0.01$ ) [48].

2. Effects of age, skeletal maturity, curve magnitude, and exercise compliance with SSE in reducing Cobb angle.

Two studies investigated the relationship between age and intervention effects [40,41]. One RCT with high study quality (PEDro: 9) revealed that in the SEAS group, participants aged $\geq 13$ years had better results than younger patients $\left(-5.8^{\circ}\right.$ vs $\left.-4.8^{\circ}\right)$ [41]. One CCT with moderate study quality (PEDro: 6) revealed the opposite result in terms of better outcomes $(-6.8 \pm 5.5 \%$ $3.1 \pm 4.2^{\circ}$ vs $\left.-1.5 \pm 4.8^{\circ}\right)$, favoring younger patients $(<13$ years) [40].

One study analyzed the interaction effect of skeletal maturity, in the form of Risser sign, with SSE in improving scoliotic deformity [40]. This study suggested that subjects with Risser stage 0 significantly benefited from
SSE in curve regression compared with those with Risser stage 3 (Risser stage 0: $5.7 \pm 5.6^{\circ}$ vs Risser stage 3: $2.1 \pm$ $\left.4.7^{\circ}\right)$.

Two studies compared decreasing values in Cobb angle between thoracic and lumbar curves (Table 3). One study demonstrated that only body awareness therapy could significantly improve thoracic curvatures, and yet, no difference was detected in comparison with the traditional exercise group [46]. Another study revealed that both thoracic and lumbar Cobb angles decreased in all participants wearing braces regardless of the exercise strategy (SEAS vs Core muscle training) [47].

No study investigated the correlation of exercise compliance with intervention effects. Five studies reported exercise compliance in the percentage value of the prescribed dosage (Table 3: $58 \pm 0.27$ to $88 \%$ ) [38, 45-48], of which three trials reported significant intergroup differences in Cobb angles that were beyond measurement error [38, 46, 48]: one study showed greater Cobb angle reduction in patients undergoing brace with exercise [38]; another study showed that bracing was superior to exercise alone for moderate scoliosis [48]; and the third study suggested body awareness exercise with bracing could effectively improve scoliosis [46].

\section{Discussion}

This review aimed to estimate the effect of SSE on scoliotic deformity improvement. Unlike the previous reviews $[33,51,52]$, besides reporting a reduction in Cobb angle, our review emphasized the true effect in terms of reductions beyond clinical measurement errors. The clinical standard for individual curve regression was reported to be $>5^{\circ}$ [55]. Therefore, any change within or equal to $5^{\circ}$ was not considered as a true improvement. The most updated meta-analysis revealed that few RCTs can be used for effect size estimation [33], of which only three SSE studies (two with a low risk of bias [41, 45] and one with a high risk of bias [35]) showed a mean reduction of only $5^{\circ}$ (D-value of Cobb: $-8.95^{\circ},-1.05^{\circ}$ ); three studies showed greater reductions but with a high risk of bias [56-58]. Moreover, these three studies adopted no typical SSE (core muscle training [56], posture education [57], and traditional exercise therapy [58]) to compare with untreated control therapies. Hence, this metaanalysis concluded that only low-quality evidence is available to suggest that SSE improves spinal deformity [33]. Regarding the lack of studies to perform metaanalysis, our study comprehensively reviewed the most recent trials to estimate the effectiveness and clinical importance of SSE in reducing Cobb angle.

According to our review, five studies with moderate to high quality (PEDro: 6-9) reported a significant decrease in Cobb angle beyond $5^{\circ}$ (Table 3). Three studies involved patients with moderate scoliosis, and thus, 
bracing was included as an intervention strategy. Specifically, two studies adopted underarm orthosis [38, 46], and one study did not report brace type [48]. Nonetheless, two of them consistently suggested that bracing with SSE was superior to bracing alone or with traditional exercise to treat moderate scoliosis $[38,46]$. Another study implied that SSE could not replace bracing to treat moderate scoliosis due to the lack of comparable effects between the two methods [48]. However, none of the studies reported the initial in-brace correction. Therefore, the results can be challenged if the baseline in-brace correction is not evenly distributed. Additionally, a study demonstrated that SSE reduced correction loss during the bracing period [59], which indicated that the role of SSE during bracing was maintaining in-brace correction. However, without reporting the initial inbrace correction, it is not possible to determine whether SSE enhanced or maintained in-brace correction. Therefore, the role of SSE during the bracing period requires further study. Two studies considered as moderate to high quality (PEDro: 6-9) in this review compared SSE alone with traditional care for mild scoliosis [40, 41]. They consistently suggested that SSE had significant effects on a curve regression for mild scoliosis until skeletal maturity. This is promising as curves $<30^{\circ}$ are unlikely to progress after skeletal maturity [5]. Therefore, the findings of these two studies are of clinical value and encouraging for patients with mild scoliosis to commence SSE. Consequently, due to the limited number of eligible studies available, insufficient evidence is available to prove the effect of SSE on curve regression for mild scoliosis.

For secondary outcomes, five trials compared truncal asymmetry pre- and post-exercise, of which three studies consistently found that SSE was not superior to brace or other exercises in improving either ATR or shoulder balance for brace-wearing patients [38, 47, 48]. SSE was only effective for improving truncal asymmetry in patients with moderate scoliosis if used as a supplement to body awareness exercise [46]. Improvement of ATR was noted if applied to patients with only mild scoliosis [41]. In particular, two studies adopted the Posterior Trunk Symmetry Index and the Walter Reed Visual Assessment Scale $[46,47]$. One study used the angle of trunk inclination and trunk appearance perception with quantifying shoulder balance [48], and two studies used ATR only $[38,41]$ to quantify changes in trunk asymmetry. Therefore, insufficient evidence is available to support the effects of SSE on truncal asymmetry improvement. In addition, a standardized algorithm is ideal for evaluating trunk asymmetry.

Six studies evaluated QoL pre- and post-interventions (Table 3). This review revealed a high initial score in all studies, which is consistent with a previous meta- analysis [51]. Thus, investigating the effects of SSE on each domain is valuable for providing a clear understanding of where the effects lie, which can assist physiotherapists in determining which strategy should be implemented to achieve specific goals. Four trials in this review studied one domain each [38, 46-48]. Two studies were conducted by Yagci et al.; they showed that the pain domain only improved with core muscle training exercise during bracing [46, 47]. Two studies [38, 48] similarly revealed that better QoL outcomes relied on either functional or mental domains (Table 3). Hence, insufficient evidence is available to append the benefits of SSE in improving QoL.

The influencing factors for brace treatment were inbrace correction, skeletal maturity, curve magnitude, and brace compliance [60]. However, this remains undefined for SSE treatment. Therefore, this is the first review to estimate the interactions between SSE and these factors. This is clinically valuable for physiotherapists to set individualized exercise protocols and estimate prognosis for patients undertaking SSE. However, only four studies addressed this concern. One study in particular revealed that better Cobb angle reduction was achieved in patients aged $\geq 13$ years [41], whereas a recent study revealed the opposite result [40]. This inconsistency may be explained by different exercise approaches and varied acceptance of SSE in different countries. Additionally, it was intriguing that $34.3 \%(n=34)$ of the patients with atypical AIS (10 left thoracic, 4 right lumbar, and 20 left thoracic with right lumbar) were recruited in that recent study [40]. Therefore, the result should be interpreted with caution when demonstrating the effects of SSE on the AIS population. One study investigated the relationship of skeletal maturity and intervention effects and suggested that better outcomes occur in patients with early Risser stages [40]. One study found that body awareness exercise with bracing is better at correcting thoracic curves. However, this study ended prematurely at the tenth week, which is a limitation because the curve can deteriorate again after a short follow-up [26]. Up to $50 \%$ of the studies in this review reported exercise compliance with a percentage value of the prescribed dosage. Although all mentioned articles consistently state that exercise adherence is crucial, no information was available to evaluate the interactions between compliance and SSE outcomes. This knowledge gap must be addressed in the future.

The main limitation of the review is the lack of highquality studies, which makes it difficult to extract adequate data to reach any firm conclusions. The previous systematic reviews $[33,52]$, analyzed the same five studies $[35,37,41,45,48]$ included in our review, revealed a significant heterogeneity by statistical testing and concluded that no pooled effect sizes could be reliably 
reported. In addition, our review included five more updated clinical trials that showed notable methodological heterogeneity: one CCT was conducted with a large sample size $(n=99)$ but no comparative untreated controls [40]; four studies, including one CCT [38] and three RCTs [36, 46, 47], were conducted with small sample sizes $(n<50)$ which could mask variations and build up of systematic errors. Moreover, those four studies $[36,38,46,47]$ in which all included bracing strategy, introduced confounding effects of bracing and SSE in treating AIS. Therefore, considering the notable heterogeneity of enrolled studies, a meta-analysis was not performed in this review. However, this review suggested that SSE has a significant effect on Cobb angle reduction, which concurred with previous reviews [34, 52]. Additionally, this review has implications for researchers identifying knowledge gaps in this field. More RCTs are required to clarify the role of SSE as a treatment for moderate AIS during bracing. In addition, the best SSE type for different curve types as well as the most effective protocol (frequency and intensity) among those available should be determined. Moreover, the key factors that influence the success of exercise treatment should be elucidated. To achieve this goal, multicenter studies with matched groups of participants are required in the future.

\section{Conclusions}

Limited evidence with moderate quality suggested that SSE can significantly reduce Cobb angle and improve truck asymmetry. The effect of SSE with brace wearing on treating moderate scoliosis is unclear. Insufficient evidence is available to implicate any effects of SSE on changes in QoL. This is the first review to study the factors influencing the success of SSE treatment, which remains undefined and requires further investigation.

\section{Supplementary information}

Supplementary information accompanies this paper at https://doi.org/10. 1186/s12891-020-03517-6.

Additional file 1 Appendix 1: Search strategy.

\section{Abbreviations}

AIS: Adolescent idiopathic scoliosis; SSE: Scoliosis-specific scoliosis; QoL: Quality of life; SRS: Scoliosis research society; SEAS: Scientific exercise approach to scoliosis; POTSI: Posterior Trunk Symmetry Index; WRVS: Walter Reed Visual Assessment Scale; TAPS: Trunk Appearance Perception Scale; ATR: Angle of trunk rotation; ATI: Angle of trunk inclination

\section{Acknowledgements}

Nil

\section{Authors' contributions}

YF and JPYC designed the study. YF and QR searched and reviewed articles. YF drafted the manuscript. MKTT, JPYC supervised the study and obtained funding support. JPYC performed the final edits. All authors read and approved the submitted version of the manuscript.

\section{Funding}

This study was financialupported by the Sanming Project of Medicine (SZSM201612055) "Team of Excellence in Spinal Deformities and Spinal Degeneration Diseases".

\section{Availability of data and materials}

Data sharing is not applicable to this article as no datasets were generated or analysed during the current study.

Ethics approval and consent to participate

Not applicable.

Consent for publication

Not applicable.

\section{Competing interests}

The authors declare that they have no conflict of interest.

\section{Author details}

${ }^{1}$ Department of Orthopaedics and Traumatology, The University of Hong Kong - Shenzhen Hospital, 1 Haiyuan 1st Road, Futian District, Shenzhen, Guangdong, China. ${ }^{2}$ Department of Physiotherapy, The University of Hong Kong-Shenzhen Hospital, Shenzhen, Guangdong province, China.

${ }^{3}$ Department of Orthopaedics and Traumatology, The University of Hong Kong, 5/F Professional Block, Queen Mary Hospital, Pokfulam, Hong Kong SAR, China.

Received: 30 May 2020 Accepted: 20 July 2020

Published online: 27 July 2020

\section{References}

1. Weinstein SL, Dolan LA, Cheng JC, Danielsson A, Morcuende JA. Adolescent idiopathic scoliosis. Lancet. 2008;371(9623):1527-37.

2. Konieczny MR, Senyurt H, Krauspe R. Epidemiology of adolescent idiopathic scoliosis. J Child Orthop. 2013:7(1):3-9.

3. Sanders AE, Andras LM, lantorno SE, Hamilton A, Choi PD, Skaggs DL. Clinically significant psychological and emotional distress in $32 \%$ of adolescent idiopathic scoliosis patients. Spine Deform. 2018;6(4):435-40.

4. Theroux J, Stomski N, Hodgetts CJ, Ballard A, Khadra C, Le May S, et al. Prevalence of low back pain in adolescents with idiopathic scoliosis: a systematic review. Chiropr Man Therap. 2017;25:10.

5. Weinstein SL. The Natural History of Adolescent Idiopathic Scoliosis. J Pediatr Orthop. 2019;39(Issue 6, Supplement 1 Suppl 1):S44-S6.

6. Kim W, Porrino JA, Hood KA, Chadaz TS, Klauser AS, Taljanovic MS. Clinical evaluation, imaging, and Management of Adolescent Idiopathic and Adult Degenerative Scoliosis. Curr Probl Diagn Radiol. 2019;48(4):402-14.

7. Gomez JA, Hresko MT, Glotzbecker MP. Nonsurgical Management of Adolescent Idiopathic Scoliosis. J Am Academy Orthopaedic Surg. 2016; 24(8):555-64.

8. Cheung JPY, Cheung PWH, Luk KD. When should we wean bracing for adolescent idiopathic scoliosis? Clin Orthop Relat Res. 2019;477(9):2145-57.

9. Cheung JPY, Cheung PWH, Samartzis D, Luk KD. Curve progression in adolescent idiopathic scoliosis does not match skeletal growth. Clin Orthop Relat Res. 2018;476(2):429-36.

10. Cheung JP, Cheung PW, Samartzis D, Cheung KM, Luk KD. The use of the distal radius and ulna classification for the prediction of growth: peak growth spurt and growth cessation. Bone Joint J. 2016;98-B(12):1689-96.

11. van den Bogaart M, van Royen BJ, Haanstra TM, de Kleuver M, Faraj SSA. Predictive factors for brace treatment outcome in adolescent idiopathic scoliosis: a best-evidence synthesis. Eur Spine J. 2019;28(3):511-25.

12. Thompson RM, Hubbard EW, Jo CH, Virostek D, Karol LA. Brace success is related to curve type in patients with adolescent idiopathic scoliosis. J Bone Joint Surg Am. 2017;99(11):923-8.

13. Cheung JPY, Cheung PWH, Yeng WC, Chan LCK. Does curve regression occur during underarm bracing in patients with adolescent idiopathic scoliosis? Clin Orthop Relat Res. 2020;478(2):334-45.

14. Cheung JPY, Cheung PWH. Supine flexibility predicts curve progression for patients with adolescent idiopathic scoliosis undergoing underarm bracing. Bone Joint J. 2020;102-B(2):254-60. 
15. Cheung JPY, Yiu KKL, Vidyadhara S, Chan PPY, Cheung PWH, Mak KC. Predictability of supine radiographs for determining in-brace correction for adolescent idiopathic scoliosis. Spine (Phila Pa 1976). 2018;43(14):971-6.

16. Weinstein SL, Dolan LA, Wright JG, Dobbs MB. Effects of bracing in adolescents with idiopathic scoliosis. N Engl J Med. 2013;369(16):1512-21.

17. Cheung JPY, Chong CHW, Cheung PWH. Underarm bracing for adolescent idiopathic scoliosis leads to flatback deformity: the role of sagittal spinopelvic parameters. Bone Joint J. 2019;101-b(11):1370-8.

18. Wong $\mathrm{CKH}$, Cheung PWH, Luo N, Lin J, Cheung JPY. Responsiveness of EQ 5D youth version 5-level (EQ-5D-5L-Y) and 3-level (EQ-5D-3L-Y) in patients with idiopathic scoliosis. Spine (Phila Pa 1976). 2019;44(21):1507-14.

19. Wong CKH, Cheung PWH, Luo N, Cheung JPY. A head-to-head comparison of five-level (EQ-5D-5L-Y) and three-level EQ-5D-Y questionnaires in paediatric patients. Eur J Health Econ. 2019;20(5):647-56.

20. Cheung PWH, Wong CKH, Cheung JPY. An insight into the health-related quality of life of adolescent idiopathic scoliosis patients who are braced, observed, and previously braced. Spine (Phila Pa 1976). 2019;44(10):E596605.

21. Cheung PWH, Wong CKH, Lau ST, Cheung JPY. Responsiveness of the EuroQoL 5-dimension (EQ-5D) in adolescent idiopathic scoliosis. Eur Spine J. 2018:27(2):278-85.

22. Wong CKH, Cheung PWH, Samartzis D, Luk KD, Cheung KMC, Lam CLK, et al. Mapping the SRS-22r questionnaire onto the EQ-5D-5L utility score in patients with adolescent idiopathic scoliosis. PLoS One. 2017;12(4):e0175847.

23. Cheung PWH, Wong CKH, Samartzis D, Luk KDK, Lam CLK, Cheung KMC, et al. Psychometric validation of the EuroQoL 5-dimension 5-level (EQ-5D$5 \mathrm{~L}$ ) in Chinese patients with adolescent idiopathic scoliosis. Scoliosis Spinal Disord. 2016;11:19.

24. Karol LA, Virostek D, Felton K, Wheeler L. Effect of compliance counseling on brace use and success in patients with adolescent idiopathic scoliosis. Bone Joint Surg Am. 2016;98(1):9-14.

25. Negrini S, Carabalona R. Social acceptability of treatments for adolescent idiopathic scoliosis: a cross-sectional study. Scoliosis. 2006;1:14.

26. Negrini S, Donzelli S, Aulisa AG, Czaprowski D, Schreiber S, de Mauroy JC, et al. 2016 SOSORT guidelines: orthopaedic and rehabilitation treatment of idiopathic scoliosis during growth. Scoliosis Spinal Disord. 2018;13:3.

27. Bialek M, M'hango A. "FITS"concept: functional individual therapy of Scoliosis. Stud Health Technol Inform. 2008;135:250.

28. Dobosiewicz K, Durmala J, Kotwicki T. Dobosiewicz method physiotherapy for idiopathic scoliosis. Stud Health Technol Inform. 2008;135:228-36.

29. Jelačić M, Villagrasa M, Pou E, Quera-Salvá G. Rigo MJS. Barcelona Scoliosis Physical Therapy School-BSPTS-based on classical Schroth principles: short term effects on back asymmetry in idiopathic scoliosis. 2012;7(1):057.

30. Lehnert-Schroth C. Schroth's three dimensional treatment of scoliosis. ZFA (Stuttgart). 1979;55(34):1969-76.

31. Maruyama T, Takeshita K, Kitagawa T. Side-shift exercise and hitch exercise. Stud Health Technol Inform. 2008;135:246-9.

32. Romano M, Negrini A, Parzini S, Tavernaro M, Zaina F, Donzelli S, et al. SEAS (scientific exercises approach to scoliosis): a modern and effective evidence based approach to physiotherapic specific scoliosis exercises. Scoliosis. 2015; 10:3.

33. Thompson JY, Williamson EM, Williams MA, Heine PJ, Lamb SE. Effectiveness of scoliosis-specific exercises for adolescent idiopathic scoliosis compared with other non-surgical interventions: a systematic review and metaanalysis. Physiotherapy. 2019;105(2):214-34.

34. Berdishevsky H, Lebel VA, Bettany-Saltikov J, Rigo M, Lebel A, Hennes A, et al. Physiotherapy scoliosis-specific exercises - a comprehensive review of seven major schools. Scoliosis Spinal Disord. 2016;11:20.

35. Kim G, Hwangbo PN. Effects of schroth and pilates exercises on the cobb angle and weight distribution of patients with scoliosis. J Phys Ther Sci. 2016:28(3):1012-5.

36. Kim M-J, Park D-S. The effect of Schroth's three-dimensional exercises in combination with respiratory muscle exercise on Cobb's angle and pulmonary function in patients with idiopathic scoliosis. Physical Therapy Rehabilitation Science. 2017;6(3):113-9.

37. Kuru T, Yeldan I, Dereli EE, Ozdincler AR, Dikici F, Colak I. The efficacy of three-dimensional Schroth exercises in adolescent idiopathic scoliosis: a randomised controlled clinical trial. Clin Rehabil. 2016; 30(2):181-90.

38. Kwan KYH, Cheng ACS, Koh HY, Chiu AYY, Cheung KMC. Effectiveness of Schroth exercises during bracing in adolescent idiopathic scoliosis: Results from a preliminary study-SOSORT Award 2017 Winner. Scoliosis Spinal Disord. 2017;12(1)

39. Lee $W$-J, Ko Y-M, Park J-W. Effect of trunk side shift exercise on the cobb\&rsquo;s angle of patients with idiopathic scoliosis. The Journal of Korean Physical Therapy. 2017;29(5):276-80.

40. Liu D, Huang S, Yu X, Yang J, Xuan X, Yang J, et al. Effects of Specific Exercise Therapy on Adolescent Patients with Idiopathic Scoliosis: A Prospective Controlled Cohort Study. Spine (Phila Pa 1976). 2020.

41. Monticone M, Ambrosini E, Cazzaniga D, Rocca B, Ferrante S. Active selfcorrection and task-oriented exercises reduce spinal deformity and improve quality of life in subjects with mild adolescent idiopathic scoliosis. Results of a randomised controlled trial. Eur Spine J. 2014;23(6):1204-14.

42. Negrini S, Donzelli S, Lusini M, Minnella S, Zaina F. The effectiveness of combined bracing and exercise in adolescent idiopathic scoliosis based on SRS and SOSORT criteria: a prospective study. BMC Musculoskelet Disord. 2014;15(1):263.

43. Schreiber S, Parent EC, Hill DL, Hedden DM, Moreau MJ, Southon SC. Schroth physiotherapeutic scoliosis-specific exercises for adolescent idiopathic scoliosis: How many patients require treatment to prevent one deterioration? - results from a randomized controlled trial - "SOSORT 2017 Award Winner". Scoliosis Spinal Disord. 2017;12(1)

44. Schreiber S, Parent EC, Hill DL, Hedden DM, Moreau MJ, Southon SC. Patients with adolescent idiopathic scoliosis perceive positive improvements regardless of change in the Cobb angle - Results from a randomized controlled trial comparing a 6-month Schroth intervention added to standard care and standard care alone. SOSORT 2018 Award winner. BMC Musculoskelet Disord. 2019;20(1):319.

45. Schreiber S, Parent EC, Khodayari Moez E, Hedden DM, Hill DL, Moreau M, et al. Schroth physiotherapeutic scoliosis-specific exercises added to the standard of care Lead to better cobb angle outcomes in adolescents with idiopathic scoliosis - an Assessor and statistician blinded randomized controlled trial. PLoS One. 2016;11(12):e0168746.

46. Yagci G, Ayhan C, Yakut Y. Effectiveness of basic body awareness therapy in adolescents with idiopathic scoliosis: a randomized controlled study1. J Back Musculoskelet Rehabil. 2018;31(4):693-701.

47. Yagci G, Yakut Y. Core stabilization exercises versus scoliosis-specific exercises in moderate idiopathic scoliosis treatment. Prosthetics Orthot Int. 2019;43(3):301-8.

48. Zheng Y, Dang Y, Yang Y, Li H, Zhang L, Lou EHM, et al. Whether orthotic management and exercise are equally effective to the patients with adolescent idiopathic scoliosis in mainland China?: a randomized controlled trial study. Spine (Phila Pa 1976). 2018;43(9):E494-e503.

49. Tolo VT, Herring JA. Scoliosis-specific exercises: a state of the art review. Spine Deform. 2020;8(2):149-55.

50. Denton DA. National Health and Medical Research Council (NHMRC) 2009; 2009

51. Burger M, Coetzee W, du Plessis LZ, Geldenhuys L, Joubert F, Myburgh E, et al. The effectiveness of Schroth exercises in adolescents with idiopathic scoliosis: a systematic review and meta-analysis. S Afr J Physiother. 2019; 75(1):904

52. Day JM, Fletcher J, Coghlan M, Ravine T. Review of scoliosis-specific exercise methods used to correct adolescent idiopathic scoliosis. Arch Physiother. 2019;9:8.

53. de Morton NA. The PEDro scale is a valid measure of the methodological quality of clinical trials: a demographic study. Australia J Physiotherapy. 2009;55(2):129-33.

54. Moseley AM, Rahman P, Wells GA, Zadro JR, Sherrington C, Toupin-April K, et al. Agreement between the Cochrane risk of bias tool and physiotherapy evidence database (PEDro) scale: a meta-epidemiological study of randomized controlled trials of physical therapy interventions. PLoS One. 2019;14(9):e0222770.

55. Richards BS, Bernstein RM, D'Amato CR, Thompson GH. Standardization of criteria for adolescent idiopathic scoliosis brace studies: SRS Committee on bracing and nonoperative management. Spine (Phila Pa 1976). 2005;30(18): 2068-75 discussion 76-7.

56. Gur G, Ayhan C, Yakut Y. The effectiveness of core stabilization exercise in adolescent idiopathic scoliosis: a randomized controlled trial. Prosthetics Orthot Int. 2017:41(3):303-10.

57. Toledo P, de Mello D, Araújo M, Daoud R, Dantas EH. Global posture reeducation effects in students with scoliosis. Fisioterapia e Pesquisa. 2011; 18(4):329-34. 
58. Wan LJZLK. Exercise therapy in treatment of essential S-shaped scoliosis: evaluation of Cobb angle in breast and lumbar segment through a followup of half a year. 2005;9:82-4.

59. Zaina F, Negrini S, Atanasio S, Fusco C, Romano M, Negrini AJS. Specific exercises performed in the period of brace weaning can avoid loss of correction in Adolescent Idiopathic Scoliosis (AIS) patients: Winner of SOSORT's 2008 Award for Best Clinical Paper. 2009;4(1):8.

60. Lo YF, Huang YC. Bracing in adolescent idiopathic scoliosis. Hu Li Za Zhi. 2017;64(2):117-23.

\section{Publisher's Note}

Springer Nature remains neutral with regard to jurisdictional claims in published maps and institutional affiliations.

Ready to submit your research? Choose BMC and benefit from:

- fast, convenient online submission

- thorough peer review by experienced researchers in your field

- rapid publication on acceptance

- support for research data, including large and complex data types

- gold Open Access which fosters wider collaboration and increased citations

- maximum visibility for your research: over $100 \mathrm{M}$ website views per year

At $\mathrm{BMC}$, research is always in progress.

Learn more biomedcentral.com/submissions 